\title{
Level-One Representations and Vertex Operators of Quantum Affine Superalgebra $U_{q}[g l(\widehat{N \mid} N)]$
}

\author{
Yao-Zhong Zhang \\ Department of Mathematics, University of Queensland, Brisbane, Qld 4072, Australia \\ Email: yzz@maths.uq.edu.au
}

\begin{abstract}
Level-one representations of the quantum affine superalgebra $U_{q}[g l(\widehat{N \mid} N)]$ associated to the appropriate non-standard system of simple roots and $q$-vertex operators (intertwining operators) associated with the level-one modules are constructed explicitly in terms of free bosonic fields.
\end{abstract}




\section{Introduction}

The algebraic analysis approach [1, 2] based on quantum affine algebra symmetries enables one not only to solve massive or off-critical integrable models directly in the thermodynamic limit but also to compute their correlation functions [3] and form factors [4] in the form of integrals by applying the techniques similar to those used so successfully in the critical cases (see, c.f. [5]). The key components behind this method are infinite dimensional highest weight representations of the quantum affine algebras and the corresponding $q$-vertex operators [6] which are intertwiners of these representations. As in the critical cases, this procedure requires the explicit construction of the highest weight representations and vertex operators in terms of free bosonic fields.

By now, the level-one representations and vertex operators have been constructed in terms of free bosons for most quantum affine bosonic algebras (see, e.g. [7, 8, 9, 10, 11, 12]). In contrast, much less has been known for the case of quantum affine superalgebras. For the type I quantum affine superalgebra $U_{q}[g l(\widehat{M \mid} N)], M \neq N$, the level-one representations and vertex operators have been investigated in [13] (see [14] for a level- $k$ free boson realization of $\left.\left.U_{q}[s l \widehat{(2 \mid} 1)\right]\right)$. In particular, the level-one irreducible highest weight representations of $\left.U_{q}[g l \widehat{l(2 \mid} 1)\right]$ were studied in some details and the correpsonding characters were derived [13]. These representations have been re-examined and used to compute the correlation functions of the $q$-deformed supersymmetric $t$ - $J$ model in [15].

So far in the literature, the very interesting case of $M=N$ has been largely ignored. The only exception is [16] where the special case of $M=N=2$ was treated and the type I vertex operators involving infinite dimensional evaluation (or level-zero) representations were also constructed for this special case. By contrast, we shall consider the general $M=N$ case and investigate both type I and type II vertex operators with respect to finite dimensional evaluation modules. The $M=N$ case is interesting since it seems to us that $U_{q}[g l(\widehat{N \mid} N)]$ is the only untwisted superalgebra which has a non-standard system where all simple roots are odd or fermionic. It also seems to be the only superalgebra where a vertex type quasi-Hopf twistor can be constructed [17 and thus the correpsonding elliptic quantum supergroup $\mathcal{A}_{q, p}[g l(\widehat{N \mid} N)]$ can be introduced.

In this paper, we construct a level-one representation of $U_{q}[g l(\widehat{N \mid} N)]$ by bosonizing the Drinfeld generators. We also construct the vertex operators associated with the level-one representations in terms of the free bosonic fields.

The layout of this paper is the following. In section 2, we describe the Drinfeld realization 18 of $U_{q}[g l(\widehat{N \mid} N)]$ in the non-standard system of simple roots and determine 
the "main terms" [19] in the coproduct formulae of the Drinfeld generators. In section 3, we derive the $2 N$-dimensional evaluation (or level-zero) representations of $U_{q}[g l(\widehat{N \mid} N)]$. In section 4, we investigate the bosonization of $U_{q}[g l(\widehat{N \mid} N)]$ and construct an explicit level-one representation in terms of free bosonic fields. Section 5 is devoted to the study of the bosonization of the level-one vertex operators.

\section{Quantum Affine Superalgebra $U_{q}[g l(\widehat{N \mid} N)]$}

As is well-known, a given Kac-Moody superalgebra 20 allows many inequivalent systems of simple roots. A system of simple roots is called distinguished if it has minimal odd roots. Let $\left\{\alpha_{i}, i=0,1, \cdots, 2 N-1\right\}$ denote a chosen set of simple roots of the affine superalgebra $g l(\widehat{N \mid} N)$. Let $($, ) be a fixed invariant bilinear form on the root space. Let $\mathcal{H}$ be the Cartan subalgebra and throughout we identify the dual $\mathcal{H}^{*}$ with $\mathcal{H}$ via (, ). As is shown in [17], $g l(\widehat{N \mid} N)$ has a simple root system in which all simple roots are odd (or fermionic). This system can be constructed from the distinguished simple root system by using the "extended" Weyl operation [21] repeatedly. We have the following simple roots, all of which are odd (or fermionic)

$$
\begin{aligned}
& \alpha_{0}=\delta-\varepsilon_{1}+\varepsilon_{2 N}, \\
& \alpha_{l}=\varepsilon_{l}-\varepsilon_{l+1}, \quad l=1,2, \cdots, 2 N-1
\end{aligned}
$$

with $\delta,\left\{\varepsilon_{k}\right\}_{k=1}^{2 N}$ satisfying

$$
(\delta, \delta)=\left(\delta, \varepsilon_{k}\right)=0, \quad\left(\varepsilon_{k}, \varepsilon_{k^{\prime}}\right)=(-1)^{k+1} \delta_{k k^{\prime}}
$$

Such a simple root system is usually called non-standard. The generalized symmetric Cartan matrix $\left(a_{i i^{\prime}}\right)$ takes the form

$$
\begin{aligned}
& a_{01} \equiv\left(\alpha_{0}, \alpha_{1}\right)=-1, \quad a_{0,2 N-1} \equiv\left(\alpha_{0}, \alpha_{2 N-1}\right)=1, \\
& a_{l l^{\prime}} \equiv\left(\alpha_{l}, \alpha_{l^{\prime}}\right)=(-1)^{l+1}\left(\delta_{l, l^{\prime}-1}-\delta_{l, l^{\prime}+1}\right), \quad l, l^{\prime}=1,2, \cdots, 2 N-1 .
\end{aligned}
$$

This Cartan matrix is degenrate. To obtain a non-degenerate Cartan matrix, we extend 22 $\mathcal{H}$ by adding to it the element

$$
\alpha_{2 N}=\sum_{k=1}^{2 N} \varepsilon_{k} .
$$

In the following, we denote by $\tilde{\mathcal{H}}$ the extended Cartan subalgebra and by $\tilde{\mathcal{H}}^{*}$ the dual of $\tilde{\mathcal{H}}$. The enlarged Cartan matrix has the following extra matrix elements:

$$
a_{2 N, 2 N} \equiv\left(\alpha_{2 N}, \alpha_{2 N}\right)=0, \quad a_{i, 2 N} \equiv\left(\alpha_{i}, \alpha_{2 N}\right)=2 \cdot(-1)^{i+1}
$$


Let $\left\{h_{0}, h_{1}, \cdots, h_{2 N}, d\right\}$ be a basis of $\tilde{\mathcal{H}}$, where $h_{2 N}$ is the element in $\tilde{\mathcal{H}}$ corresponding to $\alpha_{2 N}$ and $d$ is the usual derivation operator. We shall write $h_{i}=\alpha_{i}(i=0,1, \cdots, 2 N)$ with $\alpha_{i}$ given by ([1.1, [1.4). Let $\left\{\Lambda_{0}, \Lambda_{1}, \cdots, \Lambda_{2 N}, c\right\}$ be the dual basis with $\Lambda_{j}$ being fundamental weights and $c$ the canonical central element. We have [17]

$$
\begin{aligned}
& \Lambda_{2 N}=\frac{1}{2 N} \sum_{k=1}^{2 N}(-1)^{k+1} \varepsilon_{k} \\
& \Lambda_{i}=d+\sum_{k=1}^{i}(-1)^{k+1} \varepsilon_{k}-\frac{i}{2 N} \sum_{k=1}^{2 N}(-1)^{k+1} \varepsilon_{k},
\end{aligned}
$$

where $i=0,1, \cdots, 2 N-1$.

The quantum affine superalgebra $U_{q}[g l(\widehat{N \mid} N)]$ is a quantum (or $q^{-}$) deformation of the universal enveloping algebra of $g l(\widehat{N \mid} N)$ and is generated by the Chevalley generators $\left\{e_{i}, f_{i} q^{h_{j}}, d \mid i=0,1, \cdots, 2 N-1, j=0,1, \cdots, 2 N\right\}$. The $\mathbf{Z}_{2}$-grading of the Chevalley generators is $\left[e_{i}\right]=\left[f_{i}\right]=1, i=0,1, \cdots, 2 N-1$ and zero otherwise. The defining relations are

$$
\begin{aligned}
& h h^{\prime}=h^{\prime} h, \quad \forall h \in \tilde{\mathcal{H}}, \\
& q^{h_{j}} e_{i} q^{-h_{j}}=q^{a_{i j}} e_{i}, \quad\left[d, e_{i}\right]=\delta_{i 0} e_{i}, \\
& q^{h_{j}} f_{i} q^{-h_{j}}=q^{-a_{i j}} f_{i}, \quad\left[d, f_{i}\right]=-\delta_{i 0} f_{i}, \\
& {\left[e_{i}, f_{i^{\prime}}\right]=\delta_{i i^{\prime}} \frac{q^{h^{h}}-q^{-h_{i}}}{q-q^{-1}},} \\
& {\left[e_{i}, e_{i^{\prime}}\right]=\left[f_{i}, f_{i^{\prime}}\right]=0, \quad \text { for } \quad a_{i i^{\prime}}=0,} \\
& {\left[\left[e_{0}, e_{1}\right]_{q^{-1}},\left[e_{0}, e_{2 N-1}\right]_{q}\right]=0,} \\
& {\left[\left[e_{l}, e_{l-1}\right]_{q^{(-1)}},\left[e_{l}, e_{l+1}\right]_{q^{(-1)}}{ }^{l+1}\right]=0,} \\
& {\left[\left[e_{2 N-1}, e_{2 N-2}\right]_{q^{-1}},\left[e_{2 N-1}, e_{0}\right]_{q}\right]=0,} \\
& {\left[\left[f_{0}, f_{1}\right]_{q^{-1}},\left[f_{0}, f_{2 N-1}\right]_{q}\right]=0,} \\
& {\left[\left[f_{l}, f_{l-1}\right]_{q^{(-1)}},\left[f_{l}, f_{l+1}\right]_{q^{(-1)}}{ }^{l+1}\right]=0,} \\
& {\left[\left[f_{2 N-1}, f_{2 N-2}\right]_{q^{-1}},\left[f_{2 N-1}, f_{0}\right]_{q}\right]=0, \quad l=1,2, \cdots, 2 N-2 .}
\end{aligned}
$$

Here and throughout, $[a, b]_{x} \equiv a b-(-1)^{[a][b]} x b a$ and $[a, b] \equiv[a, b]_{1}$. The four-th order $q$-Serre relations are obtained by using Yamane's Dynkin diagram procedure [23].

$U_{q}[\operatorname{gl}(\widehat{N \mid} N)]$ is a $\mathbf{Z}_{2}$-graded quasi-triangular Hopf algebra endowed with the following coproduct $\Delta$, counit $\epsilon$ and antipode $S$ :

$$
\begin{aligned}
\Delta(h) & =h \otimes 1+1 \otimes h, \\
\Delta\left(e_{i}\right) & =e_{i} \otimes 1+q^{h_{i}} \otimes e_{i}, \quad \Delta\left(f_{i}\right)=f_{i} \otimes q^{-h_{i}}+1 \otimes f_{i}, \\
\epsilon\left(e_{i}\right) & =\epsilon\left(f_{i}\right)=\epsilon(h)=0, \\
S\left(e_{i}\right) & =-q^{-h_{i}} e_{i}, \quad S\left(f_{i}\right)=-f_{i} q^{h_{i}}, \quad S(h)=-h,
\end{aligned}
$$


where $i=0,1, \cdots, 2 N-1$ and $h \in \tilde{\mathcal{H}}$. Notice that the antipode $S$ is a $\mathbf{Z}_{2}$-graded algebra anti-homomorphism. Namely, for any homogeneous elements $a, b \in U_{q}[g l(\widehat{N \mid} N)]$ $S(a b)=(-1)^{[a][b]} S(b) S(a)$, which extends to inhomogeneous elements through linearity. Moreover,

$$
S^{2}(a)=q^{-2 \rho} a q^{2 \rho}, \quad \forall a \in U_{q}[g l(\widehat{N \mid} N)]
$$

where $\rho$ is an element in $\tilde{\mathcal{H}}$ such that $\left(\rho, \alpha_{i}\right)=\left(\alpha_{i}, \alpha_{i}\right) / 2$ for any simple root $\alpha_{i}, i=$ $0,1,2, \cdots, 2 N-1$. Explicitly,

$$
\rho=\frac{1}{2} \sum_{k=1}^{2 N}(-1)^{k} \varepsilon_{k},
$$

which coincides with $\bar{\rho}$, the half-sum of positive roots of $g l(N \mid N)$ in the present simple root system. The multiplication rule on the tensor products is $\mathbf{Z}_{2}$-graded: $(a \otimes b)\left(a^{\prime} \otimes b^{\prime}\right)=$ $(-1)^{[b]\left[a^{\prime}\right]}\left(a a^{\prime} \otimes b b^{\prime}\right)$ for any homogeneous elements $a, b, a^{\prime}, b^{\prime} \in U_{q}[g l(\widehat{N \mid} N)]$. We also introduce the element in $\tilde{\mathcal{H}}$

$$
\tilde{\rho}=\sum_{i=0}^{2 N-1} \Lambda_{i}+\xi N \Lambda_{2 N},
$$

which gives the principal gradation

$$
\left[\tilde{\rho}, e_{i}\right]=e_{i}, \quad\left[\tilde{\rho}, f_{i}\right]=-f_{i}, \quad i=0,1, \cdots, 2 N-1
$$

In (III.11), $\xi$ is an arbitrary constant.

$U_{q}[g l(\widehat{N \mid} N)]$ can also be realized in terms of the Drinfeld generators [18] $\left\{X_{m}^{ \pm, i}, H_{n}^{j}, q^{ \pm H_{0}^{j}}\right.$, $c, d \mid m \in \mathbf{Z}, n \in \mathbf{Z}-\{0\}, i=1,2, \cdots, 2 N-1, j=1,2, \cdots, 2 N\}$. The $\mathbf{Z}_{2}$-grading of the Drinfeld generators is given by $\left[X_{m}^{ \pm, i}\right]=1$ for all $i=1, \cdots, 2 N-1, m \in \mathbf{Z}$ and $\left[H_{n}^{j}\right]=\left[H_{0}^{j}\right]=[c]=[d]=0$ for all $j=1, \cdots, 2 N, n \in \mathbf{Z}-\{0\}$. The relations satisfied by the Drinfeld generators read (see [23, 24] for the Drinfeld realization of $U_{q}[g l(\widehat{N \mid} N)]$ in the distinguished system of simple roots)

$$
\begin{aligned}
& {[c, a]=\left[d, H_{0}^{j}\right]=\left[H_{0}^{j}, H_{n}^{j^{\prime}}\right]=0, \quad \forall a \in U_{q}[g l(\widehat{N \mid} N)]} \\
& q^{H_{0}^{j}} X_{n}^{ \pm, i} q^{-H_{0}^{j}}=q^{ \pm a_{i j}} X_{n}^{ \pm, i} \\
& {\left[d, X_{n}^{ \pm, i}\right]=n X_{n}^{ \pm, i}, \quad\left[d, H_{n}^{j}\right]=n H_{n}^{j},} \\
& {\left[H_{n}^{j}, H_{m}^{j^{\prime}}\right]=\delta_{n+m, 0} \frac{\left[a_{j j^{\prime}} n\right]_{q}[n c]_{q}}{n},} \\
& {\left[H_{n}^{j}, X_{m}^{ \pm, i}\right]= \pm \frac{\left[a_{i j} n\right]_{q}}{n} X_{n+m}^{ \pm, i} q^{\mp|n| c / 2}} \\
& {\left[X_{n}^{+, i}, X_{m}^{-, i^{\prime}}\right]=\frac{\delta_{i i^{\prime}}}{q-q^{-1}}\left(q^{\frac{c}{2}(n-m)} \psi_{n+m}^{+, i}-q^{-\frac{c}{2}(n-m)} \psi_{n+m}^{-, i}\right),} \\
& {\left[X_{n}^{ \pm, i}, X_{m}^{ \pm, i^{\prime}}\right]=0, \quad \text { for } a_{i i^{\prime}}=0} \\
& {\left[X_{n+1}^{ \pm, i}, X_{m}^{ \pm, i^{\prime}}\right]_{q^{ \pm a} i i^{\prime}}-\left[X_{m+1}^{ \pm, i^{\prime}}, X_{n}^{ \pm, i}\right]_{q^{ \pm a} i i^{\prime}}=0,} \\
& {\left[\left[X_{m}^{ \pm, l}, X_{m^{\prime}}^{ \pm, l-1}\right]_{q^{(-1)}},\left[X_{n}^{ \pm, l}, X_{n^{\prime}}^{ \pm, l+1}\right]_{q^{(-1)}} l+1\right]}
\end{aligned}
$$




$$
+\left[\left[X_{n}^{ \pm, l}, X_{m^{\prime}}^{ \pm, l-1}\right]_{q^{(-1)}},\left[X_{m}^{ \pm, l}, X_{n^{\prime}}^{ \pm, l+1}\right]_{q^{(-1)}}{ }^{l+1}\right]=0, \quad l=2, \cdots, 2 N-2 .
$$

where $[x]_{q}=\left(q^{x}-q^{-x}\right) /\left(q-q^{-1}\right)$ and $\psi_{n}^{ \pm, j}$ are related to $H_{ \pm n}^{j}$ by relations

$$
\sum_{n \in \mathbf{Z}} \psi_{n}^{ \pm, j} z^{-n}=q^{ \pm H_{0}^{j}} \exp \left( \pm\left(q-q^{-1}\right) \sum_{n>0} H_{ \pm n}^{j} z^{\mp n}\right)
$$

The following relations can be proved by induction:

$$
\begin{aligned}
H_{n}^{j} & =\frac{1}{q-q^{-1}} \sum_{p_{1}+2 p_{2}+\cdots+n p_{n}=n} \frac{(-1)^{\sum p_{i}-1}\left(\sum p_{i}-1\right) !}{p_{1} ! \cdots p_{n} !}\left(q^{-H_{0}^{j}} \psi_{1}^{+, j}\right)^{p_{1}} \cdots\left(q^{-H_{0}^{j}} \psi_{n}^{+, j}\right)^{p_{n}}, \\
H_{-n}^{j} & =\frac{1}{q^{-1}-q} \sum_{p_{1}+2 p_{2}+\cdots+n p_{n}=n} \frac{(-1)^{\sum p_{i}-1}\left(\sum p_{i}-1\right) !}{p_{1} ! \cdots p_{n} !}\left(q^{H_{0}^{j}} \psi_{-1}^{-, j}\right)^{p_{1}} \cdots\left(q^{H_{0}^{j}} \psi_{-n}^{-, j}\right)^{p(\operatorname{III} .15)}
\end{aligned}
$$

The Chevalley generators are related to the Drinfeld generators by the formulae

$$
\begin{aligned}
& h_{i}=H_{0}^{i}, \quad e_{i}=X_{0}^{+, i}, \quad f_{i}=X_{0}^{-, i}, \quad i=1,2, \cdots, 2 N-1, \\
& h_{2 N}=H_{0}^{2 N}, \quad h_{0}=c-\sum_{k=1}^{2 N-1} H_{0}^{k}, \\
& e_{0}=\left[X_{0}^{-, 2 N-1},\left[X_{0}^{-, 2 N-2}, \cdots,\left[X_{0}^{-, 3},\left[X_{0}^{-, 2}, X_{1}^{-, 1}\right]_{q}\right]_{q^{-1}} \cdots\right]_{q}\right]_{q^{-1}} q^{-\sum_{k=1}^{2 N-1} H_{0}^{k}}, \\
& f_{0}=(-1)^{N} q^{\sum_{k=1}^{2 N-1} H_{0}^{k}}\left[\left[\cdots\left[\left[X_{-1}^{+, 1}, X_{0}^{+, 2}\right]_{q^{-1}} X_{0}^{+, 3}\right]_{q}, \cdots, X_{0}^{+, 2 N-2}\right]_{q^{-1}}, X_{0}^{+, 2 N-1}\right](\mathrm{II}
\end{aligned}
$$

The coproduct of the Drinfeld generators is not known in full. However, for our purpose it suffices to derive the "main terms" [19] in the coproduct formulae. We have Proposition 1 : For $m \in \mathbf{Z}_{\geq 0}, n \in \mathbf{Z}_{>0}$ and $i=1,2, \cdots, 2 N-1$,

$$
\begin{aligned}
\Delta\left(X_{m}^{+, i}\right)= & X_{m}^{+, i} \otimes q^{m c}+q^{H_{0}^{i}+2 m c} \otimes X_{m}^{+, i} \\
& +\sum_{k=0}^{m-1} q^{\frac{1}{2}(m+3 k) c} \psi_{m-k}^{+, i} \otimes q^{(m-k) c} X_{k}^{+, i} \bmod N_{-} \otimes N_{+}^{2}, \\
\Delta\left(X_{-n}^{+, i}\right)= & X_{-n}^{+, i} \otimes q^{-n c}+q^{-H_{0}^{i}} \otimes X_{-n}^{+, i} \\
& +\sum_{k=1}^{n-1} q^{\frac{1}{2}(n-k) c} \psi_{k-n}^{-, i} \otimes q^{(k-n) c} X_{-n}^{+, i} \bmod N_{-} \otimes N_{+}^{2}, \\
\Delta\left(X_{n}^{-, i}\right)= & X_{n}^{-, i} \otimes q^{H_{0}^{i}}+q^{n c} \otimes X_{n}^{-, i} \\
& +\sum_{k=1}^{n-1} q^{(n-k) c} X_{k}^{-, i} \otimes q^{\frac{1}{2}(k-n) c} \psi_{n-k}^{+, i} \bmod N_{-}^{2} \otimes N_{+}, \\
\Delta\left(X_{-m}^{-, i}\right)= & X_{-m}^{-, i} \otimes q^{-H_{0}^{i}-2 m c}+q^{-m c} \otimes X_{-m}^{-, i} \\
& +\sum_{k=0}^{m-1} q^{(k-m) c} X_{-k}^{-, i} \otimes q^{-\frac{1}{2}(m+3 k) c} \psi_{k-m}^{-, i} \bmod N_{-}^{2} \otimes N_{+}, \\
\Delta\left(H_{n}^{i}\right)= & H_{n}^{i} \otimes q^{\frac{1}{2} n c}+q^{\frac{3}{2} n c} \otimes H_{n}^{i} \bmod N_{-} \otimes N_{+}, \\
\Delta\left(H_{-n}^{i}\right)= & H_{-n}^{i} \otimes q^{-\frac{3}{2} n c}+q^{-\frac{1}{2} n c} \otimes H_{-n}^{i} \bmod N_{-} \otimes N_{+},
\end{aligned}
$$

where $N_{ \pm}$and $N_{ \pm}^{2}$ are the left ideals generated by $X_{l}^{ \pm, k}$ and $X_{l}^{ \pm, k} X_{l^{\prime}}^{ \pm, k^{\prime}}, k, k^{\prime}=1, \cdots, 2 N-$ $1 ; l, l^{\prime} \in \mathbf{Z}$, respectively. 
Remark. (i) We do not write down the formulae for $\Delta\left(H_{ \pm n}^{2 N}\right)$ because they are not needed in this paper. $\Delta\left(H_{ \pm n}^{2 N}\right)$ can be determined by requiring that $\Delta$ preserves the commutation relations ([1.13). (ii) Modulo $N_{+} \otimes N_{-}+N_{-} \otimes N_{+}$, the elements $\psi_{ \pm n}^{ \pm, i}(n \geq 0)$ are group-like:

$$
\begin{aligned}
& \Delta\left(\psi_{n}^{+, i}\right)=\sum_{k=0}^{n} q^{\frac{3}{2} k c} \psi_{n-k}^{+, i} \otimes q^{\frac{1}{2}(n-k) c} \psi_{k}^{+, i}, \\
& \Delta\left(\psi_{-n}^{-, i}\right)=\sum_{k=0}^{n} q^{-\frac{1}{2} k c} \psi_{k-n}^{-, i} \otimes q^{\frac{3}{2}(k-n) c} \psi_{-k}^{-, i} .
\end{aligned}
$$

Define the Drinfeld currents or generating functions,

$$
X^{ \pm, i}(z)=\sum_{n \in \mathbf{Z}} X_{n}^{ \pm, i} z^{-n-1}, \quad \psi^{ \pm, j}(z)=\sum_{n \in \mathbf{Z}} \psi_{n}^{ \pm, j} z^{-n}
$$

In terms of these currents, (ㅍ.13) read

$$
\begin{aligned}
& \psi^{ \pm, j}(z) \psi^{ \pm, j^{\prime}}(w)=\psi^{ \pm, j^{\prime}}(w) \psi^{ \pm, j}(z), \\
& \psi^{+, j}(z) \psi^{-, j^{\prime}}(w)=\frac{\left(z-w q^{c+a_{j j^{\prime}}}\right)\left(z-w q^{-c-a_{j j^{\prime}}}\right)}{\left(z-w q^{c-a_{j j^{\prime}}}\right)\left(z-w q^{\left.-c+a_{j j^{\prime}}\right)}\right.} \psi^{-, j^{\prime}}(w) \psi^{+, j}(z), \\
& \psi^{+, j}(z) X^{ \pm, i}(w)=q^{ \pm a_{i j}} \frac{z-w q^{\mp \frac{c}{2} \mp a_{i j}}}{z-w q^{\mp \frac{c}{2} \pm a_{i j}}} X^{ \pm, i}(w) \psi^{+, j}(z), \\
& \psi^{-, j}(z) X^{ \pm, i}(w)=q^{ \pm a_{i j}} \frac{z-w q^{ \pm \frac{c}{2} \mp a_{i j}}}{z-w q^{ \pm \frac{c}{2} \pm a_{i j}}} X^{ \pm, i}(w) \psi^{-, j}(z), \\
& {\left[X^{+, i}(z), X^{-, i^{\prime}}(w)\right]=\frac{\delta_{i i^{\prime}}}{\left(q-q^{-1}\right) z w}\left(\delta\left(\frac{w}{z} q^{c}\right) \psi^{+, i}\left(w q^{\frac{c}{2}}\right)-\delta\left(\frac{w}{z} q^{-c}\right) \psi^{-, i}\left(w q^{-\frac{c}{2}}\right)\right),} \\
& X^{ \pm, i}(z) X^{ \pm, i^{\prime}}(w)+X^{ \pm, i^{\prime}}(w) X^{ \pm, i}(z)=0, \quad \text { for } a_{i i^{\prime}}=0, \\
& \left(z-w q^{ \pm a_{i i^{\prime}}}\right) X^{ \pm, i}(z) X^{ \pm, i^{\prime}}(w)+\left(z q^{ \pm a_{i i^{\prime}}}-w\right) X^{ \pm, i^{\prime}}(w) X^{ \pm, i}(z)=0, \\
& \left\{\left[\left[X^{ \pm, l}\left(z_{1}\right), X^{ \pm, l-1}(z)\right]_{q^{(-1)}},\left[X^{ \pm, l}\left(z_{2}\right), X^{ \pm, l+1}(w)\right]_{q^{(-1)^{l}+1}}\right]\right\} \\
& +\left\{z_{1} \leftrightarrow z_{2}\right\}=0, \quad l=2,3, \cdots, 2 N-2 .
\end{aligned}
$$

These current commutation relations can be derived from the super version [24, 25] of the RS algebra [26] by means of the Gauss decomposition technique of Ding and Frenkel [27].

\section{Level-Zero Representation}

We consider the evaluation representation $V_{z}$ of $U_{q}[g l(\widehat{N \mid} N)]$, where $V$ is an $2 N$-dimensional graded vector space with basis vectors $\left\{v_{1}, v_{2}, \cdots, v_{2 N}\right\}$. The $\mathbf{Z}_{2}$-grading of the basis vectors is chosen to be $\left[v_{j}\right]=\frac{(-1)^{j}+1}{2}$. Let $e_{j, j^{\prime}}$ be the $2 N \times 2 N$ matrix satisfying $\left(e_{j, j^{\prime}}\right)_{k k^{\prime}}=\delta_{j k} \delta_{j^{\prime} k^{\prime}}$ or equivalently $e_{i, j} v_{k}=\delta_{j k} v_{i}$, (which implies that for any operator $A$ its matrix elements $A_{j, i}$ are defined by $A v_{i}=A_{j, i} v_{j}$ ). In the homogeneous gradation, 
the Chevalley generators on $V_{z}$ are represented by

$$
\begin{aligned}
& e_{i}=e_{i, i+1}, \quad f_{i}=(-1)^{i+1} e_{i+1, i}, \quad i=1,2, \cdots, 2 N-1, \\
& h_{i}=(-1)^{i+1}\left(e_{i, i}+e_{i+1, i+1}\right), \quad h_{2 N}=\sum_{k=1}^{2 N}(-1)^{k+1} e_{k, k} \\
& e_{0}=z e_{2 N, 1}, \quad f_{0}=-z^{-1} e_{1,2 N}, \quad h_{0}=-e_{1,1}-e_{2 N, 2 N} .
\end{aligned}
$$

Let $V^{* S}$ be the left dual module of $V$, defined by

$$
\left(a \cdot v^{*}\right)(v)=(-1)^{[a]\left[v^{*}\right]} v^{*}(S(a) v), \quad \forall a \in U_{q}[g l(\widehat{N \mid} N)], v \in V, v^{*} \in V^{*}
$$

Namely, the representations on $V^{* S}$ are given by

$$
\pi_{V^{* S}}(a)=\pi_{V}(S(a))^{s t}, \quad \forall a \in U_{q}[g l(\widehat{N \mid} N)]
$$

where $s t$ denotes the supertansposition defined by $\left(A_{i, j}\right)^{s t}=(-1)^{[j]([i]+[j])} A_{j, i}$. Note that in general $\left.\left(\left(A_{i, j}\right)^{s t}\right)\right)^{s t}=(-1)^{[A]} A_{i, j} \neq A_{i, j}$. Let $V_{z}^{* S}$ be the $2 N$-dimensional evaluation module corresponding to $V^{* S}$. On $V_{z}^{* S}$, the Chevalley generators are represented by

$$
\begin{aligned}
& e_{i}=-(-1)^{i} q^{(-1)^{i}} e_{i+1, i}, \quad f_{i}=-q^{(-1)^{i+1}} e_{i, i+1}, \quad i=1,2, \cdots, 2 N-1, \\
& h_{i}=(-1)^{i}\left(e_{i, i}+e_{i+1, i+1}\right), \quad h_{2 N}=\sum_{k=1}^{2 N}(-1)^{k} e_{k, k} \\
& e_{0}=z q e_{1,2 N}, \quad f_{0}=z^{-1} q^{-1} e_{2 N, 1}, \quad h_{0}=e_{1,1}+e_{2 N, 2 N} .
\end{aligned}
$$

Proposition 2 : The Drinfeld generators are represented on $V_{z}$ by

$$
\begin{aligned}
H_{m}^{i}= & (-1)^{i+1} \frac{[m]_{q}}{m} q^{(-1)^{i} m}\left(q^{x_{i}} z\right)^{m}\left(e_{i, i}+e_{i+1, i+1}\right), \\
H_{m}^{2 N}= & z^{m} \frac{[2 m]_{q}}{m}\left[-q^{m} \sum_{l=1}^{N} e_{2 l, 2 l}\right. \\
& \left.+\sum_{l=1}^{N}\left(y+(l-1)\left(1-q^{m}\right)\right)\left(e_{2 l-1,2 l-1}+e_{2 l, 2 l}\right)\right], \\
H_{0}^{i}= & (-1)^{i+1}\left(e_{i, i}+e_{i+1, i+1}\right), \quad H_{0}^{2 N}=\sum_{k=1}^{2 N}(-1)^{k+1} e_{k, k}, \\
X_{m}^{+, i}= & \left(q^{x_{i}} z\right)^{m} e_{i, i+1}, \quad X_{m}^{-, i}=(-1)^{i+1}\left(q^{x_{i}} z\right)^{m} e_{i+1, i},
\end{aligned}
$$

and on $V_{z}^{* S}$ by

$$
\begin{aligned}
H_{m}^{i} & =(-1)^{i} \frac{[m]_{q}}{m} q^{(-1)^{i+1} m}\left(q^{-x_{i}} z\right)^{m}\left(e_{i, i}+e_{i+1, i+1}\right) \\
H_{m}^{2 N} & =-z^{m} \frac{[2 m]_{q}}{m}\left[-q^{-m} \sum_{l=1}^{N} e_{2 l, 2 l}\right.
\end{aligned}
$$


Zhang: Level-One Representations and Vertex Operators of $U_{q}[g l(\widehat{N \mid} N)]$

$$
\begin{gathered}
\left.+\sum_{l=1}^{N}\left(-y^{*}+(l-1)\left(1-q^{-m}\right)\right)\left(e_{2 l-1,2 l-1}+e_{2 l, 2 l}\right)\right] \\
H_{0}^{i}=(-1)^{i}\left(e_{i, i}+e_{i+1, i+1}\right), \quad H_{0}^{2 N}=\sum_{k=1}^{2 N}(-1)^{k} e_{k, k}, \\
X_{m}^{+, i}=-(-1)^{i} q^{(-1)^{i}}\left(q^{-x_{i}} z\right)^{m} e_{i+1, i}, \quad X_{m}^{-, i}=-q^{(-1)^{i+1}}\left(q^{-x_{i}} z\right)^{m} e_{i, i+1},
\end{gathered}
$$

where $i=1, \cdots, 2 N-1, x_{i}=\sum_{l=1}^{i}(-1)^{l+1}=\frac{(-1)^{i+1}+1}{2}$ and $y, y^{*}$ are arbitrary constants.

\section{Free Boson Realization at Level One}

We use the notations similar to those in 9, 13. Let us introduce bosonic oscillators $\left\{A_{n}^{j}, c_{n}^{l}, Q_{A^{j}}, Q_{c^{l}} \mid n \in \mathbf{Z}, j=1,2, \cdots, 2 N, l=1,2, \cdots, N\right\}$ which satisfy the commutation relations

$$
\begin{aligned}
& {\left[A_{n}^{j}, A_{m}^{j^{\prime}}\right]=\delta_{n+m, 0} \frac{\left[a_{j j^{\prime}} n\right]_{q}[n]_{q}}{n}, \quad\left[A_{0}^{j}, Q_{A^{j^{\prime}}}\right]=a_{j j^{\prime}}} \\
& {\left[c_{n}^{l}, c_{m}^{l^{\prime}}\right]=\delta_{l l^{\prime}} \delta_{n+m, 0} \frac{[n]_{q}^{2}}{n}, \quad\left[c_{0}^{l}, Q_{c^{l^{\prime}}}\right]=\delta_{l l^{\prime}} .}
\end{aligned}
$$

The remaining commutation relations are zero. Introduce the currents

$$
\begin{aligned}
& H^{j}(z ; \kappa)=Q_{A^{j}}+A_{0}^{j} \ln z-\sum_{n \neq 0} \frac{A_{n}^{j}}{[n]_{q}} q^{\kappa|n|} z^{-n}, \\
& c^{l}(z)=Q_{c^{l}}+c_{0}^{l} \ln z-\sum_{n \neq 0} \frac{c_{n}^{l}}{[n]_{q}} z^{-n}
\end{aligned}
$$

and set

$$
\begin{aligned}
H_{ \pm}^{j}(z) & =H^{j}\left(q^{ \pm \frac{1}{2}} z ;-\frac{1}{2}\right)-H^{j}\left(q^{\mp \frac{1}{2}} z ; \frac{1}{2}\right) \\
& = \pm\left(q-q^{-1}\right) \sum_{n>0} A_{ \pm n}^{j} z^{\mp n} \pm A_{0}^{i} \ln q .
\end{aligned}
$$

We make a basis transformation and express $A_{n}^{j}$ and $Q_{A^{j}}$ in terms of a new set of bosonic oscillators $\left\{a_{n}^{j}, Q_{a^{j}} \mid j=1,2, \cdots, 2 N\right\}$ as

$$
\begin{aligned}
& A_{n}^{i}=(-1)^{i+1}\left(a_{n}^{i}+a_{n}^{i+1}\right), \quad A_{n}^{2 N}=\frac{q^{n}+q^{-n}}{2} \sum_{l=1}^{2 N}(-1)^{l+1} a_{n}^{l}, \\
& Q_{A^{i}}=(-1)^{i+1}\left(Q_{a^{i}}+Q_{a^{i+1}}\right), \quad Q_{A^{2 N}}=\sum_{l=1}^{2 N}(-1)^{l+1} Q_{a^{l}},
\end{aligned}
$$

where $i=1, \cdots, 2 N-1$ and $\left\{a_{n}^{j}, Q_{a^{j}}\right\}$ satisfy the commutation relations

$$
\left[a_{n}^{j}, a_{m}^{j^{\prime}}\right]=(-1)^{j+1} \delta_{j j^{\prime}} \delta_{n+m, 0} \frac{[n]_{q}^{2}}{n}, \quad\left[a_{0}^{j}, Q_{a^{j^{\prime}}}\right]=(-1)^{j+1} \delta_{j j^{\prime}} .
$$

Now we state our main result in this section on the free boson realization of $U_{q}[g l(\widehat{N \mid} N)]$ at level one. 
Theorem 1 : The Drinfeld generators of $U_{q}[g l(\widehat{N \mid} N)]$ at level one are realized by the free boson fields as

$$
\begin{aligned}
& c=1, \\
& \psi^{ \pm, j}(z)=e^{H_{ \pm}^{j}(z)}, \quad j=1,2, \cdots, 2 N, \\
& X^{ \pm, i}(z)=: e^{ \pm H^{i}\left(z ; \mp \frac{1}{2}\right)} Y^{ \pm, i}(z): F^{ \pm, i}, \quad i=1,2, \cdots, 2 N-1,
\end{aligned}
$$

where

$$
\begin{aligned}
& F^{ \pm, 2 k-1}=\prod_{l=1}^{k-1} e^{ \pm \sqrt{-1} \pi a_{0}^{2 l-1}}, \quad F^{ \pm, 2 k}=\prod_{l=1}^{k} e^{\mp \sqrt{-1} \pi a_{0}^{2 l-1}}, \\
& Y^{+, 2 k-1}(z)=e^{c^{k}(z)}, \\
& Y^{-, 2 k-1}(z)=\frac{1}{z\left(q-q^{-1}\right)}\left(e^{-c^{k}(q z)}-e^{-c^{k}\left(q^{-1} z\right)}\right), \\
& Y^{+, 2 k}(z)=Y^{-, 2 k-1}(z)=\frac{1}{z\left(q-q^{-1}\right)}\left(e^{-c^{k}(q z)}-e^{-c^{k}\left(q^{-1} z\right)}\right), \\
& Y^{-, 2 k}(z)=-Y^{+, 2 k-1}(z)=-e^{c^{k}(z)}, \quad k=1,2, \cdots, N .
\end{aligned}
$$

Proof. We prove this theorem by checking that they satisfy the defining relations (II.20) of $U_{q}[g l(\widehat{N \mid} N)]$ with $c=1$. It is easily seen that the first two relations in ([I.20) are true by construction. The third and fourth ones follow from the definition of $X^{ \pm, i}(z)$ and the commutativity between $a_{n}^{j}$ and $c_{n}^{l}$. So we only need to check the last three relations in (II.20).

We write

$$
Z^{ \pm, i}(z)=: e^{ \pm H^{i}\left(z ; \mp \frac{1}{2}\right)}: F^{ \pm, i}
$$

It is easily shown that

$$
\begin{aligned}
& Z^{+, i}(z) Z^{+, i^{\prime}}(w)=\left\{\begin{array}{l}
: Z^{+, i}(z) Z^{+, i^{\prime}}(w): \quad \text { for } a_{i i^{\prime}}=0 \text { and } i \leq i^{\prime}, \\
-: Z^{+, i}(z) Z^{+, i^{\prime}}(w): \quad \text { for } a_{i i^{\prime}}=0 \text { and } i>i^{\prime}, \\
\left(z-q^{-1} w\right): Z^{+, i}(z) Z^{+, i^{\prime}}(w): \quad \text { for } a_{i i^{\prime}}=1 \text { and } i<i^{\prime},(\mathrm{IV} .9) \\
-\left(z-q^{-1} w\right): Z^{+, i}(z) Z^{+, i^{\prime}}(w): \quad \text { for } a_{i i^{\prime}}=1 \text { and } i>i^{\prime} \\
\left(z-q^{-1} w\right)^{-1}: Z^{+, i}(z) Z^{+, i^{\prime}}(w): \quad \text { for } a_{i i^{\prime}}=-1
\end{array}\right. \\
& Z^{-, i}(z) Z^{-, i^{\prime}}(w)=\left\{\begin{array}{l}
: Z^{-, i}(z) Z^{-, i^{\prime}}(w): \quad \text { for } a_{i i^{\prime}}=0 \text { and } i \leq i^{\prime}, \\
-: Z^{-, i}(z) Z^{-, i^{\prime}}(w): \quad \text { for } a_{i i^{\prime}}=0 \text { and } i>i^{\prime} \\
(z-q w): Z^{-, i}(z) Z^{-, i^{\prime}}(w): \quad \text { for } a_{i i^{\prime}}=1 \text { and } i<i^{\prime}, \text { (IV.10) } \\
-(z-q w): Z^{-, i}(z) Z^{-, i^{\prime}}(w): \quad \text { for } a_{i i^{\prime}}=1 \text { and } i>i^{\prime}, \\
(z-q w)^{-1}: Z^{-, i}(z) Z^{-, i^{\prime}}(w): \quad \text { for } a_{i i^{\prime}}=-1,
\end{array}\right.
\end{aligned}
$$




$$
Z^{+, i}(z) Z^{-, i^{\prime}}(w)=\left\{\begin{array}{l}
: Z^{+, i}(z) Z^{-, i^{\prime}}(w): \quad \text { for } a_{i i^{\prime}}=0 \text { and } i \leq i^{\prime} \\
-: Z^{+, i}(z) Z^{-, i^{\prime}}(w): \quad \text { for } a_{i i^{\prime}}=0 \text { and } i>i^{\prime}, \\
(z-w)^{-1}: Z^{+, i}(z) Z^{-, i^{\prime}}(w): \quad \text { for } a_{i i^{\prime}}=1 \text { and } i<i^{\prime},(\text { IV.11) } \\
-(z-w)^{-1}: Z^{+, i}(z) Z^{-, i^{\prime}}(w): \quad \text { for } a_{i i^{\prime}}=1 \text { and } i>i^{\prime}, \\
(z-w): Z^{+, i}(z) Z^{-, i^{\prime}}(w): \text { for } a_{i i^{\prime}}=-1,
\end{array}\right.
$$

We have similar formulae for $Z^{+, i^{\prime}}(w) Z^{+, i}(z), Z^{-, i^{\prime}}(w) Z^{-, i}(z)$ and $Z^{-, i^{\prime}}(w) Z^{+, i}(z)$.

We now compute operator products $Y^{+, i}(z) Y^{+, i^{\prime}}(w)$ and $Y^{+, i}(z) Y^{-, i^{\prime}}(w)$. It is easily seen from the definition of $Y^{ \pm, i}(z)$ that the non-trivial products are those corresponding to $i=i^{\prime}$ and $a_{i i^{\prime}}=1$. Note that $a_{i i^{\prime}}=1$ whenever $i=2 k-1, i^{\prime}=2 k\left(\right.$ or $\left.i=2 k, i^{\prime}=2 k-1\right)$ where $k=1,2, \cdots, N-1$. The corresponding operator products are

$$
\begin{aligned}
& Y^{+, 2 k-1}(z) Y^{+, 2 k}(w)=\frac{1}{w\left(q-q^{-1}\right)}\left(\frac{: e^{c^{k}(z)} e^{-c^{k}(q w)}:}{z-q w}-\frac{: e^{c^{k}(z)} e^{-c^{k}\left(q^{-1} w\right)}:}{z-q^{-1} w}\right), \\
& Y^{-, 2 k-1}(z) Y^{-, 2 k}(w)=-\frac{1}{z\left(q-q^{-1}\right)}\left(\frac{: e^{-c^{k}(q z)} e^{c^{k}(w)}:}{q z-w}-\frac{: e^{-c^{k}\left(q^{-1} z\right)} e^{c^{k}(w)}:}{q^{-1} z-w}\right) \text {, } \\
& Y^{+, 2 k-1}(z) Y^{-, 2 k}(w)=-(z-w): Y^{+, 2 k-1}(z) Y^{-, 2 k}(w): \\
& Y^{+, 2 k}(z) Y^{-, 2 k-1}(w)=\frac{1}{z w\left(q-q^{-1}\right)}\left(q(z-w): e^{-c^{k}(q z)} e^{-c^{k}(q w)}:\right. \\
& -q^{-1}(z-w): e^{-c^{k}\left(q^{-1} z\right)} e^{-c^{k}\left(q^{-1} w\right)}: \\
& -\left(q z-q^{-1} w\right): e^{-c^{k}(q z)} e^{-c^{k}\left(q^{-1} w\right)}: \\
& \left.-\left(q^{-1} z-q w\right): e^{-c^{k}\left(q^{-1} z\right)} e^{-c^{k}(q w)}:\right) \text {. }
\end{aligned}
$$

Since $Y^{+, 2 k}(z)=Y^{-, 2 k-1}(z)$ and $Y^{-, 2 k}(z)=-Y^{+, 2 k-1}(z)$, the products $Y^{+, i}(z) Y^{+, i}(w)$ and $Y^{ \pm, i}(z) Y^{\mp, i}(w)$ can be deduced from (IV.12). For example,

$$
\begin{aligned}
Y^{+, 2 k}(z) Y^{-, 2 k}(w) & =-Y^{+, 2 k}(z) Y^{+, 2 k-1}(w) \\
& =\frac{1}{z\left(q-q^{-1}\right)}\left(\frac{: e^{-c^{k}\left(q^{-1} z\right)} e^{c^{k}(w)}:}{q^{-1} z-w}-\frac{: e^{-c^{k}(q z)} e^{c^{k}(w)}:}{q z-w}\right) .
\end{aligned}
$$

By means of (IV.9), (IV.11), (IV.10) and (IV.12) we can show that the last three relations in ([1.20) are satisfied by ([V.7). For instance,

$$
\begin{aligned}
{\left[X^{+, 2 k}(z), X^{-, 2 k-1}(w)\right]=} & -\frac{1}{z w\left(q-q^{-1}\right)^{2}}: Z^{+, 2 k}(z) Z^{-, 2 k-1}(w):\left(\frac{1}{z-w}+\frac{1}{w-z}\right) \\
& \times\left(\left(q z-q^{-1} w\right): e^{-c^{k}(q z)} e^{-c^{k}\left(q^{-1} w\right)}:\right. \\
& \left.+\left(q^{-1} z-q w\right): e^{-c^{k}\left(q^{-1} z\right)} e^{-c^{k}(q w)}:\right) \\
= & -\frac{1}{z^{2} w\left(q-q^{-1}\right)^{2}}: Z^{+, 2 k}(z) Z^{-, 2 k-1}(w): \\
& \times \delta\left(\frac{w}{z}\right)\left(\left(q z-q^{-1} w\right): e^{-c^{k}(q z)} e^{-c^{k}\left(q^{-1} w\right):}\right. \\
& \left.+\left(q^{-1} z-q w\right): e^{-c^{k}\left(q^{-1} z\right)} e^{-c^{k}(q w)}:\right)=0 .
\end{aligned}
$$




\section{Bosonization of Level-One Vertex Operators}

In this section, we study the level-one vertex operators [6] of $U_{q}[g l(\widehat{N \mid} N)]$. Let $V(\lambda)$ be the highest weight $U_{q}[g l(\widehat{N \mid} N)]$-module with the highest weight $\lambda$. Consider the following intertwiners of $U_{q}[g l(\widehat{N \mid} N)]$-modules [2]:

$$
\begin{aligned}
\Phi_{\lambda}^{\mu V}(z): & V(\lambda) \longrightarrow V(\mu) \otimes V_{z}, \\
\Phi_{\lambda}^{\mu V^{*}}(z): & V(\lambda) \longrightarrow V(\mu) \otimes V_{z}^{* S}, \\
\Psi_{\lambda}^{V \mu}(z): & V(\lambda) \longrightarrow V_{z} \otimes V(\mu), \\
\Psi_{\lambda}^{V^{*} \mu}(z): & V(\lambda) \longrightarrow V_{z}^{* S} \otimes V(\mu) .
\end{aligned}
$$

They are intertwiners in the sense that for any $x \in U_{q}[g l(\widehat{N \mid} N)]$

$$
\Xi(z) \cdot x=\Delta(x) \cdot \Xi(z), \quad \Xi(z)=\Phi_{\lambda}^{\mu V}(z), \Phi_{\lambda}^{\mu V^{*}}(z), \Psi_{\lambda}^{V \mu}(z), \Psi_{\lambda}^{V^{*} \mu}(z)
$$

These intertwiners are even operators, that is their gradings are $\left[\Phi_{\lambda}^{\mu V}(z)\right]=\left[\Phi_{\lambda}^{\mu V^{*}}(z)\right]=$ $\left[\Psi_{\lambda}^{V \mu}(z)\right]=\left[\Psi_{\lambda}^{V^{*} \mu}(z)\right]=0$. According to [2], $\Phi_{\lambda}^{\mu V}(z)\left(\Phi_{\lambda}^{\mu V^{*}}(z)\right)$ is called type I (dual) vertex operator and $\Psi_{\lambda}^{V \mu}(z)\left(\Psi_{\lambda}^{V^{*} \mu}(z)\right)$ type II (dual) vertex operator.

We expand the vertex operators as a formal series [2]

$$
\begin{array}{ll}
\Phi_{\lambda}^{\mu V}(z)=\sum_{j=1}^{2 N} \Phi_{\lambda, j}^{\mu V}(z) \otimes v_{j}, & \Phi_{\lambda}^{\mu V^{*}}(z)=\sum_{j=1}^{2 N} \Phi_{\lambda, j}^{\mu V^{*}}(z) \otimes v_{j}^{*}, \\
\Psi_{\lambda}^{V \mu}(z)=\sum_{j=1}^{2 N} v_{j} \otimes \Psi_{\lambda, j}^{V \mu}(z), & \Psi_{\lambda}^{V^{*} \mu}(z)=\sum_{j=1}^{2 N} v_{j}^{*} \otimes \Psi_{\lambda, j}^{V^{*} \mu}(z) .
\end{array}
$$

Then the intertwining property $(\overline{V .5}$ ) reads in terms of components

$$
\begin{aligned}
& \sum \Phi_{\lambda, j}^{\mu V}(z) x \otimes v_{j}(-1)^{\left[v_{j}\right][x]}=\sum x_{(1)} \Phi_{\lambda, j}^{\mu V}(z) \otimes x_{(2)} v_{j}(-1)^{\left[v_{j}\right]\left[x_{(2)}\right]}, \\
& \sum \Phi_{\lambda, j}^{\mu V^{*}}(z) x \otimes v_{j}^{*}(-1)^{\left[v_{j}^{*}\right][x]}=\sum x_{(1)} \Phi_{\lambda, j}^{\mu V^{*}}(z) \otimes x_{(2)} v_{j}^{*}(-1)^{\left[v_{j}^{*}\right]\left[x_{(2)}\right]}, \\
& \sum v_{j} \otimes \Psi_{\lambda, j}^{V \mu}(z) x=\sum x_{(1)} v_{j} \otimes x_{(2)} \Psi_{\lambda, j}^{V \mu}(z)(-1)^{\left[v_{j}\right]\left[x_{(2)}\right]}, \\
& \sum v_{j}^{*} \otimes \Psi_{\lambda, j}^{V^{*} \mu}(z) x=\sum x_{(1)} v_{j}^{*} \otimes x_{(2)} \Psi_{\lambda, j}^{V^{*} \mu}(z)(-1)^{\left[v_{j}^{*}\right]\left[x_{(2)}\right]},
\end{aligned}
$$

where we have used the notation $\Delta(x)=\sum_{x} x_{(1)} \otimes x_{(2)}$ and the fact that the vertex operators are even which implies $\left[\Phi_{\lambda, j}^{\mu V}(z)\right]=\left[\Phi_{\lambda, j}^{\mu V^{*}}(z)\right]=\left[\Psi_{\lambda, j}^{V \mu}(z)\right]=\left[\Psi_{\lambda, j}^{V^{*} \mu}(z)\right]=\left[v_{j}\right]=$ $\frac{(-1)^{j}+1}{2}$.

Introduce the even operators $\phi(z), \phi^{*}(z), \psi(z)$ and $\psi^{*}(z)$,

$$
\begin{array}{ll}
\phi(z)=\sum_{j=1}^{2 N} \phi_{j}(z) \otimes v_{j}, & \phi^{*}(z)=\sum_{j=1}^{2 N} \phi_{j}^{*}(z) \otimes v_{j}^{*} \\
\psi(z)=\sum_{j=1}^{2 N} v_{j} \otimes \psi_{j}(z), & \psi^{*}(z)=\sum_{j=1}^{2 N} v_{j}^{*} \otimes \psi_{j}^{*}(z) .
\end{array}
$$


The grading of the components is given by $\left[\phi_{j}(z)\right]=\left[\phi_{j}^{*}(z)\right]=\left[\psi_{j}(z)\right]=\left[\psi_{j}^{*}(z)\right]=\frac{(-1)^{j}+1}{2}$. Now we state

Proposition 3 : Assume that the operators $\phi(z), \phi^{*}(z), \psi(z), \psi^{*}(z)$ satisfy the intertwining relations (V.X). Then the operators $\phi(z)$ and $\psi(z)$ with respect to $V_{z}$ are determined by the components $\phi_{2 N}(z)$ and $\psi_{1}(z)$, respectively. With respect to $V_{z}^{* S}$, the operators $\phi^{*}(z)$ and $\psi^{*}(z)$ are determined by $\phi_{1}^{*}(z)$ and $\psi_{2 N}^{*}(z)$, respectively. More explicitly, we have for $l=1,2, \cdots, 2 N-1$,

$$
\begin{gathered}
(-1)^{l} \phi_{l}(z)=\left[\phi_{l+1}(z), f_{l}\right]_{q^{(-1)^{l}}}, \\
{\left[\phi_{l}(z), f_{l}\right]_{q^{(-1)^{l}}}=0,} \\
{\left[\phi_{k}(z), f_{l}\right]=0, \quad k \neq l, l+1 .} \\
q^{(-1)^{l+1}} \phi_{l+1}^{*}(z)=\left[\phi_{l}^{*}(z), f_{l}\right]_{q^{(-1)^{l+1}}}, \\
{\left[\phi_{l+1}^{*}(z), f_{l}\right]_{q^{(-1)^{l+1}}}=0,} \\
{\left[\phi_{k}^{*}(z), f_{l}\right]=0, \quad k \neq l, l+1 .} \\
{\left[\psi_{l+1}(z)=\left[\psi_{l}(z), e_{l}\right]_{q^{(-1)^{l+1}}}\right.} \\
{\left[\psi_{l+1}(z), e_{l}\right]_{q^{(-1)^{l+1}}}=0,} \\
\left.(z), e_{l}\right]=0, \quad k \neq l, l+1 . \\
{\left[\psi_{l}^{*}(z), e_{l}\right]_{q^{(-1)^{l}}}=0,} \\
{\left[\psi_{k}^{*}(z), e_{l}\right]=0, \quad k \neq l, l+1 .}
\end{gathered}
$$

Next we determine the relations of the components $\phi_{2 N}(z), \phi_{1}^{*}(z), \psi_{1}(z), \psi_{2 N}^{*}(z)$ and the Drinfeld generators. By means of proposition 1 and the intertwining relations, we have

Proposition 4 : For $\phi(z)$ associated with $V_{z}$,

$$
\begin{aligned}
& {\left[\phi_{2 N}(z), X^{+, i}(w)\right]=0,} \\
& q^{h_{i}} \phi_{2 N}(z) q^{-h_{i}}=q^{-\delta_{i, 2 N-1}} \phi_{2 N}(z), \\
& {\left[H_{n}^{i}, \phi_{2 N}(z)\right]=-\delta_{i, 2 N-1} q^{\frac{3}{2} n} \frac{[n]_{q}}{n} z^{n} \phi_{2 N}(z),} \\
& {\left[H_{-n}^{i}, \phi_{2 N}(z)\right]=-\delta_{i, 2 N-1} q^{-\frac{1}{2} n} \frac{[n]_{q}}{n} z^{-n} \phi_{2 N}(z) ;}
\end{aligned}
$$


for $\phi^{*}(z)$ associated with $V_{z}^{*}$,

$$
\begin{aligned}
& {\left[\phi_{1}^{*}(z), X^{+, i}(w)\right]=0,} \\
& q^{h_{i}} \phi_{1}^{*}(z) q^{-h_{i}}=q^{\delta_{i, 1}} \phi_{1}^{*}(z), \\
& {\left[H_{n}^{i}, \phi_{1}^{*}(z)\right]=\delta_{i, 1} q^{\frac{3}{2} n} \frac{[n]_{q}}{n} z^{n} \phi_{1}^{*}(z),} \\
& {\left[H_{-n}^{i}, \phi_{1}^{*}(z)\right]=\delta_{i, 1} q^{-\frac{1}{2} n} \frac{[n]_{q}}{n} z^{-n} \phi_{1}^{*}(z) ;}
\end{aligned}
$$

for $\psi(z)$ associated with $V_{z}$,

$$
\begin{aligned}
& {\left[\psi_{1}(z), X^{-, i}(w)\right]=0,} \\
& q^{h_{i}} \psi_{1}(z) q^{-h_{i}}=q^{-\delta_{i, 1}} \psi_{1}(z), \\
& {\left[H_{n}^{i}, \psi_{1}(z)\right]=-\delta_{i, 1} q^{\frac{1}{2} n} \frac{[n]_{q}}{n} z^{n} \psi_{1}(z),} \\
& {\left[H_{-n}^{i}, \psi_{1}(z)\right]=-\delta_{i, 1} q^{-\frac{3}{2} n} \frac{[n]_{q}}{n} z^{-n} \psi_{1}(z) ;}
\end{aligned}
$$

and for $\psi^{*}(z)$ associated with $V_{z}^{*}$,

$$
\begin{aligned}
& {\left[\psi_{2 N}^{*}(z), X^{-, i}(w)\right]=0,} \\
& q^{h_{i}} \psi_{2 N}^{*}(z) q^{-h_{i}}=q^{\delta_{i, 2 N-1}} \psi_{2 N}^{*}(z), \\
& {\left[H_{n}^{i}, \psi_{2 N}^{*}(z)\right]=\delta_{i, 2 N-1} q^{\frac{1}{2} n} \frac{[n]_{q}}{n} z^{n} \psi_{2 N}^{*}(z),} \\
& {\left[H_{-n}^{i}, \psi_{2 N}^{*}(z)\right]=\delta_{i, 2 N-1} q^{-\frac{3}{2} n} \frac{[n]_{q}}{n} z^{-n} \psi_{2 N}^{*}(z) .}
\end{aligned}
$$

In order to obtain bosonized expressions of the vertex operators, we introduce the following combinations of the Drinfeld generators:

$$
\begin{aligned}
& A_{n}^{* i}=\sum_{l=1}^{2 N-1} a_{i l}^{-1} A_{n}^{l}+\frac{2}{q^{n}+q^{-n}} a_{i, 2 N}^{-1} A_{n}^{2 N}, \\
& A_{0}^{* i}=\sum_{l=1}^{2 N} a_{i l}^{-1} A_{0}^{l}, \quad Q_{A^{i}}^{*}=\sum_{l=1}^{2 N} a_{i l}^{-1} Q_{A^{l}}, \quad i=1,2, \cdots, 2 N-1,
\end{aligned}
$$

which satisfy the relations

$$
\begin{aligned}
& {\left[A_{n}^{* i}, A_{m}^{i^{\prime}}\right]=\delta_{i i^{\prime}} \delta_{n+m, 0} \frac{[n]_{q}^{2}}{n},} \\
& {\left[A_{n}^{* i}, A_{m}^{* i^{\prime}}\right]=a_{i i^{\prime}}^{-1} \delta_{n+m, 0} \frac{[n]_{q}^{2}}{n},} \\
& {\left[A_{0}^{* i}, Q_{A^{i^{\prime}}}\right]=\delta_{i i^{\prime}}, \quad\left[A_{0}^{i}, Q_{A^{i^{\prime}}}^{*}\right]=\delta_{i i^{\prime}},} \\
& {\left[A_{0}^{* i}, Q_{A^{i^{\prime}}}^{*}\right]=a_{i i^{\prime}}^{-1}, \quad i, i^{\prime}=1,2, \cdots, 2 N-1 .}
\end{aligned}
$$

Introduce the currents,

$$
H^{*, j}(z ; \kappa)=Q_{A^{j}}^{*}+A_{0}^{* j} \ln z-\sum_{n \neq 0} \frac{A_{n}^{* j}}{[n]_{q}} q^{k|n|} z^{-n} .
$$


Now we state our main theorem in this section on the bosonic realization of the operators $\phi(z), \phi^{*}(z), \psi(z)$ and $\psi^{*}(z)$ at level one. Thanks to the previous propositions, we only need to determine one component for each operator and the other components are represented by the integral of the currents.

Theorem 2 : The components $\phi_{2 N}(z), \phi_{1}^{*}(z), \psi_{1}(z)$ and $\psi_{2 N}^{*}(z)$ can be realized explicitly as follows:

$$
\begin{aligned}
\phi_{2 N}(z)= & : e^{-H^{*, 2 N-1}\left(q z ; \frac{1}{2}\right)} e^{c^{N}(q z)}: e^{-\sqrt{-1} \pi a_{0}^{1}} \prod_{l=1}^{N-1} e^{-\sqrt{-1} \pi \frac{2 N+l}{2 N} a_{0}^{2 l+1}}, \\
\phi_{1}^{*}(z)= & : e^{H^{*, 1}\left(q z ; \frac{1}{2}\right)}: \prod_{l=1}^{N-1} e^{\sqrt{-1} \pi \frac{2 N-l}{2 N} a_{0}^{2 l+1}}, \\
\psi_{1}(z)= & : e^{-H^{*, 1}\left(q z ;-\frac{1}{2}\right)}: \prod_{l=1}^{N-1} e^{\sqrt{-1} \pi \frac{2 N-l}{2 N} a_{0}^{2 l+1}}, \\
\psi_{2 N}^{*}(z)= & \frac{1}{z\left(q-q^{-1}\right)}: e^{H^{*, 2 N-1}\left(q z ;-\frac{1}{2}\right)}\left(e^{-c^{N}\left(q^{2} z\right)}-e^{-c^{N}(z)}\right): \\
& \times e^{-\sqrt{-1} \pi a_{0}^{1}} \prod_{l=1}^{N-1} e^{-\sqrt{-1} \pi \frac{2 N+l}{2 N} a_{0}^{2 l+1}} .
\end{aligned}
$$

Proof. This theorem is proved by checking that the construction satisfies all the intertwining relations.

Remark. The following inverse elements of the extended Cartan matrix are needed to determine the cocycle factors appearing in above theorem:

$$
\begin{aligned}
& a_{2 N-1,2 l}^{-1}=a_{2 N-1,2 l+1}^{-1}=-\frac{l}{N}, \quad l=1,2, \cdots, N-1, \\
& a_{2 N-1,1}^{-1}=0, \quad a_{2 N-1,2 N}^{-1}=\frac{1}{2 N}, \\
& a_{1,2 l-1}^{-1}=a_{1,2 l}^{-1}=\frac{N-l}{N}, \quad l=1,2, \cdots, N-1, \\
& a_{1,2 N-1}^{-1}=0, \quad a_{1,2 N}^{-1}=\frac{1}{2 N} .
\end{aligned}
$$

We are now in a position to state the following result:

Proposition 5 : The vertex operators $\Phi_{\lambda}^{\mu V}(z), \Phi_{\lambda}^{\mu V^{*}}(z), \Psi_{\lambda}^{V \mu}(z)$ and $\Psi_{\lambda}^{V^{*} \mu}(z)$, if they exist, satisfy the same relations as the operators $\phi(z), \phi^{*}(z), \psi(z)$ and $\psi^{*}(z)$, respectively. This proposition follows immediately from the fact that the formers and the latters obey the same intertwining properties. Identifying $\Phi_{\lambda}^{\mu V}(z), \Phi_{\lambda}^{\mu V^{*}}(z), \Psi_{\lambda}^{V \mu}(z)$ and $\Psi_{\lambda}^{V^{*} \mu}(z)$ with $\phi(z), \phi^{*}(z), \psi(z)$ and $\psi^{*}(z)$, respectively, then the bosonic realization of the vertex operators is easily seen to be given by propositions 3 , , 4 and theorem 2 .

Acknowledgements. The financial support from Australian Research Council through a Queen Elizabeth II Fellowship Grant is gratefully acknowledged. 


\section{References}

[1] B. Davies, O. Foda, M. Jimbo, T. Miwa, A. Nakayashiki, Commun. Math. Phys.151, 89 (1993).

[2] M. Jimbo, T. Miwa, Algebraic analysis of solvable lattice models, CBMS Regional Conference Series in Mathematics, vol. 85, AMS, 1994.

[3] V.E. Korepin, N.M. Bogoliubov, A.G. Izergin, Quantum inverse scattering method and correlation functions, Cambridge University Press, 1993.

[4] F.A. Smirnov, Form factors in completely integrable models of quantum field theory, World Scientific, Singapore, 1992.

[5] C.Itzykson, H.Saleur and J.-B.Zuber, ed: Conformal invariance and applications to statistical mechanics, World Scientific, Singapore, 1988.

[6] I.B. Frenkel, N.Yu. Reshetikhin, Commun. Math. Phys. 146, 1 (1992).

[7] I.B. Frenkel, N. Jing, Proc. Nat'l. Acad. Sci. USA 85, 9373 (1988).

[8] D. Bernard, Lett. Math. Phys. 17, 239 (1989).

[9] H. Awata, S. Odake, J. Shiraishi, Commun. Math. Phys. 162, 61 (1994).

[10] Y. Koyama, Commun. Math. Phys. 164, 277 (1994).

[11] N. Jing, S.-J. Kang, Y. Koyama, Commun. Math. Phys. 174, 367 (1995).

[12] N. Jing, K.C. Misra, e-print q-alg/9701034.

[13] K. Kimura, J. Shiraishi, J. Uchiyama, Commun. Math. Phys. 188, 367 (1997).

[14] H. Awata, S. Odake, J. Shiraishi, Lett. Math. Phys. 42, 271 (1997).

[15] W.-L. Yang, Y.-Z. Zhang, Nucl. Phys. B547, 599 (1999).

[16] R. Gade, Nucl. Phys. B500, 547 (1997).

[17] Y.-Z. Zhang, M.D. Gould, e-print math.QA/9809156, J. Math. Phys., in press.

[18] V.G. Drinfeld, Sov. Math. Dokl. 36, 212 (1988).

[19] V. Chari, A. Pressley, Commun. Math. Phys. 142, 261 (1991). 
[20] V.G. Kac, Lect. Notes in Math. 676, 597 (1978).

[21] L. Frappat, A. Sciarrino, P. Sorba, Commun. Math. Phys. 121, 457 (1989).

[22] S.M. Khoroshkin, V.N. Tolstoy, Commun. Math. Phys. 141, 599 (1991).

[23] H. Yamane, e-print q-alg/9603015.

[24] Y.-Z. Zhang, J. Phys. A30, 8325 (1997).

[25] M.D. Gould, Y.-Z. Zhang, Lett. Math. Phys. 44, 291 (1998).

[26] N.Yu. Reshetikhin, M.A. Semenov-Tian-Shansky, Lett. Math. Phys. 19, 133 (1990).

[27] J. Ding, I.B. Frenkel, Commun. Math. Phys. 156, 277 (1993). 